\title{
REGULARITY OF THE BELTRAMI EQUATION AND 1-QUASICONFORMAL EMBEDDINGS OF SURFACES IN $\mathbb{R}^{3}$
}

\author{
SHANSHUANG YANG
}

\begin{abstract}
A striking result in quasiconformal mapping theory states that if $D$ is a domain in $\mathbb{R}^{n}$ (with $n \geq 3$ ) and $f: D \rightarrow \mathbb{R}^{n}$ an embedding, then $f$ is 1-QC if and only if $f$ is a Möbius transformation. This result has profound impact in quasiconformal analysis and differential geometry. This project reflects part of our effort to extend this type of rigidity results to embeddings $f: \mathbb{R}^{n} \rightarrow \mathbb{R}^{m}$ from $\mathbb{R}^{n}$ into a higher dimensional space $\mathbb{R}^{m}$ (with $m>n$ ). In this paper we focus on smooth embeddings of planar domains into $\mathbb{R}^{3}$. In particular, we show that a $C^{1+\alpha}$-smooth surface is 1-QC equivalent to a planar domain. We also show that a topological sphere that is $C^{1+\alpha}$-diffeomorphic to the standard sphere $\mathbb{S}^{2}$ is also 1-QC equivalent to $\mathbb{S}^{2}$. Along the way, a regularity result is established for solutions of the Beltrami equation with degenerate coefficient, which is used in this paper and has its own interest.
\end{abstract}

\section{INTRODUCTION}

An embedding $f: X \rightarrow Y$ in metric spaces $X$ and $Y$ is called quasiconformal, abbreviated QC, if there is a constant $K<\infty$ such that

$$
\limsup _{r \rightarrow 0} \frac{\sup \{|f(x)-f(y)|:|x-y|=r\}}{\inf \{|f(x)-f(y)|:|x-y|=r\}} \leq K
$$

for all $x \in X$. In this case we also say $f$ is $K$-QC. Here $|x-y|$ denotes the distance between $x$ and $y$ in a metric space. This type of distortion condition plays an important role in the recent development of analysis on general metric spaces (see $\mathrm{He}$ ). This paper is concerned with 1-QC maps between planar domains and surfaces.

It is well known that in the complex plane a map is 1-QC if and only if it is conformal. A classical Liouville type theorem asserts that an orientation preserving embedding of the plane $f: \mathbb{R}^{2} \rightarrow \mathbb{R}^{2}$ is 1 -QC if and only if it is a linear map: $f(z)=a z+b$. A sophisticated version of this result in higher dimensions states that if $D$ is a domain in the Euclidean space $\mathbb{R}^{n}(n \geq 3)$, then an embedding $f: D \rightarrow \mathbb{R}^{n}$ is 1-QC if and only if it is the restriction to $D$ of a Möbius transformation of $\overline{\mathbb{R}}^{n}$ (see [Ge1, Ge2] and [Re1, Re2]). A Möbius transformation is a finite composition of reflections in spheres. This deep result spells out the fundamental difference between conformal mapping theory in the plane and in higher dimensions. Its

Received by the editors August 19, 2009.

2010 Mathematics Subject Classification. Primary 30C65; Secondary 53A05, 53A30.

Key words and phrases. Quasiconformal map, conformal map, Beltrami equation, regularity.

This research was supported in part by the University Research Committee of Emory University.

(C)2009 American Mathematical Society Reverts to public domain 28 years from publication 
sophisticated proof has a long history and involves tools from analysis, geometry and PDE (see [IM1, Chapter 5] for more details). This result is also useful in differential geometry. In particular, it is a major ingredient in the proof of Mostow's rigidity on compact hyperbolic manifolds of higher dimensions $\mathbf{M 0}$.

The current project is part of our effort to extend this type of rigidity results to embeddings $f: \mathbb{R}^{n} \rightarrow \mathbb{R}^{m}$ from $\mathbb{R}^{n}$ into a higher dimensional space $\mathbb{R}^{m}$ (with $m>n$ ). In this paper we concentrate on smooth embeddings of planar domains into $\mathbb{R}^{3}$. In Section 2, we establish a regularity result for solutions of the Beltrami equation with degenerate coefficient. It will be needed in this paper and also has its own independent interest, considering the importance of the Beltrami equation. In Section 3, we give a differential geometric interpretation of 1-quasiconformality for smooth maps. Sections 4 and 5 are devoted to the two main results of this paper, Theorems 4.2 and 5.1. In Section 4, we show that the graph $\Sigma$ of any $C^{1+\alpha_{-}}$ function $\phi(x, y)$ over a domain $G$ is 1-QC equivalent to a planar domain, while Theorem 5.1 states that any surface that is $C^{1+\alpha}$-diffeomorphic to the unit sphere $\mathbb{S}^{2}$ is also 1-QC equivalent to $\mathbb{S}^{2}$. In Section 4 , we also extend Theorem 4.2 to arbitrary $C^{1+\alpha}$-smooth surfaces, not just graphs. The idea, which can be traced back to Gauss on finding isothermal coordinates for surfaces (see $\mathrm{Be}$ ), is to solve an appropriate Beltrami equation. Finally, in Section 6, we give a few examples to illustrate the main results and pose some open problems.

\section{Regularity of solutions to the Beltrami equation}

In this section we establish a regularity result for solutions of the Beltrami equation with continuous but degenerate coefficient $\mu(z)$. This extends some existing regularity results in some sense, and hence has its own interest. We also use this result to prove the 1-QC equivalence of smooth surfaces with planar domains in Section 4 .

It is well known that an orientation preserving quasiconformal mapping $f: \Omega \rightarrow$ $\mathbb{C}$ of a planar domain is a solution to the Beltrami equation

$$
\bar{\partial} f(z)=\mu(z) \partial f(z),
$$

where

$$
\bar{\partial}=\frac{\partial}{\partial \bar{z}}=\frac{1}{2}\left(\partial_{x}+i \partial_{y}\right), \quad \partial=\frac{\partial}{\partial z}=\frac{1}{2}\left(\partial_{x}-i \partial_{y}\right),
$$

and $\mu(z)$, a measurable function in $\Omega$ with $\|\mu\|_{\infty}<1$, is called the Beltrami coefficient or the complex dilatation of $f$. The main result of this section is the following.

Theorem 2.2. Let $\Omega \subset \mathbb{C}$ be an open set and $\mu$ a continuous function in $\Omega$ with $|\mu(z)|<1$ for all $z \in \Omega$. Then the Beltrami equation (2.1) has a homeomorphic solution $f: \Omega \rightarrow \mathbb{C}$ such that $f \in W_{\text {loc }}^{1,2}$. Furthermore, if the integral

$$
\int \frac{\left|\mu(z)-\mu\left(z_{0}\right)\right|}{\left|z-z_{0}\right|^{2}} d z
$$

converges uniformly in every compact subset $E$ of $\Omega$, that is, for every $\epsilon>0$ there exists a $\delta>0$ such that

$$
\int_{\left|z-z_{0}\right|<\delta} \frac{\left|\mu(z)-\mu\left(z_{0}\right)\right|}{\left|z-z_{0}\right|^{2}} d z<\epsilon
$$

for every $z_{0} \in E$, then the above solution $f$ has continuous partial derivatives of first order. 
2.4. Remarks. (a) It is easy to see that the condition (2.3) will be satisfied if $\mu$ is locally $\alpha$-Hölder continuous for some $\alpha>0$. This condition is reasonable (and in some sense necessary) in order for the solution to enjoy the continuous differentiability (see [Le, p. 28]).

(b) Theorem 2.2 extends the classical measurable Riemann mapping theorem and the regularity result $[\mathrm{LV}$, Theorem 7.2 , p. 235$]$ in the sense that the degenerate case where $\|\mu\|_{\infty}=1$ is allowed in Theorem 2.2. This also differs from the recent treatment of Iwaniec and Martin IM2 on the Beltrami equation with degenerate coefficient $\mu$. The governing assumption in [IM2] is that the corresponding distortion function

$$
K(z)=\frac{1+|\mu(z)|}{1-|\mu(z)|}
$$

is exponentially integrable. This condition does not follow from local $\alpha$-Hölder continuity of $\mu$. But the compactness argument we use here is similar to the one used in [IM2].

2.5. Proof of Theorem 2.2. Fix a decomposition of $\Omega$ :

$$
\Omega=\bigcup_{j=1}^{\infty} \Omega_{j},
$$

where $\Omega_{j}$ are open subsets of $\Omega$ with $\bar{\Omega}_{j} \subset \Omega_{j+1}$. Then, since $\mu$ is continuous, there are constants $k_{j}<1$ such that $|\mu(z)| \leq k_{j}<1$ for all $z \in \Omega_{j}$ and all $j=1,2, \ldots$. Let

$$
\mu_{j}(z)= \begin{cases}\mu(z), & \text { for } z \in \Omega_{j}, \\ 0, & \text { for } z \notin \Omega_{j} .\end{cases}
$$

We may assume that $0,1 \in \Omega_{j}$ for all $j \geq 1$. Then, by the well-known Measurable Riemann Mapping Theorem, for each $j$ there is a normalized solution $f_{j}: \mathbb{C} \rightarrow \mathbb{C}$ to the Beltrami equation with coefficient $\mu_{j}$ such that $f_{j}(0)=0, f_{j}(1)=1$ and $f_{j}(\infty)=\infty$.

We claim that, for each fixed index $n$, the family $\left\{f_{j}\right\}$ is a normal family in $\Omega_{n}$. To prove this claim, we observe that $\left\{f_{j}\right\}$ is a family of $K_{n}$-QC maps in $\Omega_{n}$ with $K_{n}=\left(1+k_{n}\right) /\left(1-k_{n}\right)<\infty$ independent of $j$ and that each $f_{j}$ omits the value $\infty$. We also note that each $f_{j}$ fixes 0 and 1 . Thus the normality condition in $[\mathrm{LV}$, Theorem 5.1, p. 73] is satisfied and it follows that $\left\{f_{j}\right\}$ is a normal family in $\Omega_{n}$. Therefore, there is a subsequence $\left\{f_{j}^{(n)}\right\}$ which converges uniformly on compact sets in $\Omega_{n}$. Using the diagonal process, we obtain a subsequence $\left\{f_{n}^{(n)}\right\}$ which converges uniformly on compact sets in $\Omega$. Denote the limit function in $\Omega$ by $f$. It follows from the compactness property of QC maps that $f$ is $K_{n}$-quasiconformal in $\Omega_{n}$ and that $f$ is injective in $\Omega$.

Next, we show that $f$ is a solution to the Beltrami equation in $\Omega$. For this we fix an arbitrary compact set $E \subset \Omega$. Then there exists index $m$ such that

$$
E \subset \Omega_{m} \subset \bar{\Omega}_{m} \subset \Omega \text {. }
$$

Thus, integrating the differential inequality

$$
\left|D f_{n}^{(n)}(z)\right|^{2} \leq K(z) J\left(z, f_{n}^{(n)}\right)
$$


for quasiconformal maps $f_{n}^{(n)}$ over $E$, we obtain that

$$
\int_{E}\left|D f_{n}^{(n)}(z)\right|^{2} d z \leq K_{m}\left|f_{n}^{(n)}(E)\right|
$$

Since the sequence $\left\{f_{n}^{(n)}\right\}$ converges uniformly on compact sets, it follows from (2.6) that the sequence $\left\{D f_{n}^{(n)}\right\}$ is locally $L^{2}$-bounded in $\Omega$. Therefore there is a subsequence, denoted again by $\left\{D f_{n}^{(n)}\right\}$, which converges weakly to $D f$ in $\Omega$. In particular, for any test function $\phi \in C_{0}^{\infty}(\Omega)$, we have

$$
\lim _{n \rightarrow \infty} \int_{\Omega}\left(\bar{\partial} f_{n}^{(n)}-\mu \partial f_{n}^{(n)}\right) \phi d z=\int_{\Omega}(\bar{\partial} f-\mu \partial f) \phi d z .
$$

Suppose $\phi$ has compact support $E \subset \Omega_{m}$ for some $m \geq 1$. Then, for large $n$, $\bar{\partial} f_{n}^{(n)}-\mu \partial f_{n}^{(n)}=\bar{\partial} f_{n}^{(n)}-\mu_{m} \partial f_{n}^{(n)}=0$ in $\Omega_{m}$. Thus it follows from (2.7) that

$$
\int_{\Omega}(\bar{\partial} f-\mu \partial f) \phi d z=0
$$

for any test function $\phi \in C_{0}^{\infty}(\Omega)$. Therefore, $f$ is a solution to the Beltrami equation $\bar{\partial} f-\mu \partial f=0$ in $\Omega$.

Finally, suppose the local uniform convergence condition (2.3) is satisfied. Applying [LV] Theorem 7.2, p. 235] to each subdomain $\Omega_{j} \subset \Omega$, we conclude that the above solution $f$ has continuous partial derivatives of first order and has the complex dilatation $\mu(z)$ for every $z \in \Omega$. This completes the proof of Theorem 2.2 .

2.8. Example. The following example shows that when $\Omega=\mathbb{C}$ the solution $f$ : $\mathbb{C} \rightarrow \mathbb{C}$ need not be onto, contrary to the nondegenerate case, but similar to the degenerate case treated in [IM2, Theorem 14.1].

Consider the radial stretching $h: \mathbb{C} \rightarrow \mathbb{C}$ given by

$$
h(z)=\rho(r) e^{i \theta}, \quad z=r e^{i \theta}, \quad \rho(r)=\frac{2}{\pi} \tan ^{-1} r .
$$

Then $h$ maps $\mathbb{C}$ onto the unit disk and satisfies the Beltrami equation $\bar{\partial} h(z)=$ $\mu(z) \partial h(z)$ with the Beltrami coefficient $\mu$ given by

$$
\begin{aligned}
\mu(z) & =\frac{r \dot{\rho}(r)-\rho(r)}{r \dot{\rho}(r)+\rho(r)} \frac{z}{\bar{z}} \\
& =\frac{r-\left(1+r^{2}\right) \tan ^{-1} r}{r+\left(1+r^{2}\right) \tan ^{-1} r} \frac{z}{\bar{z}} .
\end{aligned}
$$

It is easy to see that $\mu(z)$ is locally Hölder continuous with $|\mu(z)|<1$ for all $z \in \mathbb{C}$. Thus the Beltrami coefficient $\mu$ satisfies the conditions in Theorem 2.2. But the solution $h: \mathbb{C} \rightarrow \mathbb{C}$ is not onto.

\section{Differential geOmetric interpretations of 1-QC maps}

In this section we establish several equivalent conditions for smooth 1-QC maps on surfaces in $\mathbb{R}^{3}$ by using languages in differential geometry. By a smooth surface $\Sigma$ in $\mathbb{R}^{3}$ we mean that $\Sigma$ is a 2 -dimensional $\mathrm{C}^{1}$-manifold embedded in $\mathbb{R}^{3}$. Suppose $f: \Sigma \rightarrow \Sigma_{1}$ is a diffeomorphism between (smooth) surfaces $\Sigma$ and $\Sigma_{1}$. Then the differential of $f$ at a point $p \in \Sigma$, denoted by $d f_{p}$, is an isomorphism between the tangent vector spaces $T_{p}(\Sigma)$ and $T_{f(p)}\left(\Sigma_{1}\right)$, which are subspaces of $\mathbb{R}^{3}$. For instance, let $f=\left(f_{1}, f_{2}, f_{3}\right): D \rightarrow \Sigma_{1}$ be a diffeomorphism from a domain $D$ in $\mathbb{R}^{2}$ to a 
surface $\Sigma_{1} \subset \mathbb{R}^{3}$. Then the differential $d f_{p}$ can be represented by the following $3 \times 2$ matrix formed by the partial derivatives of the components of $f$ at $p=(x, y)$ :

$$
d f_{p}=\left(\begin{array}{ll}
\frac{\partial f_{1}(x, y)}{\partial x} & \frac{\partial f_{1}(x, y)}{\partial y} \\
\frac{\partial f_{2}(x, y)}{\partial x} & \frac{\partial f_{2}(x, y)}{\partial y} \\
\frac{\partial f_{3}(x, y)}{\partial x} & \frac{\partial f_{3}(x, y)}{\partial y}
\end{array}\right): \mathbb{R}^{2} \rightarrow \mathbb{R}^{3} .
$$

3.1. Theorem. Let $f: \Sigma \rightarrow \Sigma_{1}$ be a diffeomorphism between surfaces $\Sigma$ and $\Sigma_{1}$. Then the following conditions are equivalent.

(a) $f$ is 1-QC in the sense of definition (1.1);

(b) For all $p \in \Sigma$ and all unit vectors $e_{1}, e_{2} \in T_{p}(\Sigma)$,

$$
\left\|d f_{p}\left(e_{1}\right)\right\|=\left\|d f_{p}\left(e_{2}\right)\right\| .
$$

(c) For all $p \in \Sigma$ and all $v_{1}, v_{2} \in T_{p}(\Sigma)$ we have

$$
\left\langle d f_{p}\left(v_{1}\right), d f_{p}\left(v_{2}\right)\right\rangle=\lambda(p)^{2}\left\langle v_{1}, v_{2}\right\rangle,
$$

where $\lambda(p)=\left\|d f_{p}\right\|>0$ is the operator norm of the linear map $d f_{p}$.

(d) The intrinsic metrics $d \sigma$ on $\Sigma$ and $d \sigma_{1}$ on $\Sigma_{1}$ satisfies

$$
d \sigma_{1}^{2}=\lambda(p)^{2} d \sigma^{2}
$$

at corresponding points under the map $f$, where $\lambda(p)>0$ is the same as in (c).

3.2. Remarks. Before proving Theorem 3.1, some remarks and clarification are in order. There are two "natural" metrics on a surface $\Sigma$. One is the Euclidean metric inherited from $\mathbb{R}^{3}$ and the other is the intrinsic (or "surface") metric determined by the first fundamental form of the surface. In the quasiconformality definition (1.1), and hence in condition (a) above, we always use the Euclidean metric for subsets in an Euclidean space. The metric used in condition (d) is the intrinsic metric. Under a local parametrization $x(u, v)$ at $p \in \Sigma$, the length element $d \sigma$ of the intrinsic metric can be expressed as

$$
d \sigma^{2}=E d u^{2}+2 F d u d v+G d v^{2},
$$

where $E, F, G$ are the coefficients of the first fundamental form $I_{p}(w)=\langle w, w\rangle$ : $T_{p}(\Sigma) \rightarrow \mathbb{R}$ expressed in the basis $\left\{x_{u}, x_{v}\right\}$ associated to the parametrization $x(u, v)$. We refer the reader to $\mathrm{Ca}$. for more details on fundamental forms of surfaces and related subjects.

3.3. Proof of Theorem 3.1. For the proof of $(\mathrm{a}) \Rightarrow(\mathrm{b})$, without loss of generality, we may fix $p=0$ and assume $f(p)=0$. Given two unit vectors $e_{1}, e_{2} \in T_{0}(\Sigma)$, choose curves $\alpha$ and $\beta:(-1,1) \rightarrow \Sigma$ on $\Sigma$ with $\alpha(0)=\beta(0)=0$ such that $\alpha^{\prime}(0)=e_{1}$ and $\beta^{\prime}(0)=e_{2}$. Using local coordinates in $\mathbb{R}^{3}$, one can write $f=\left(f_{1}, f_{2}, f_{3}\right)$ and $\alpha(t)=\left(\alpha_{1}(t), \alpha_{2}(t), \alpha_{3}(t)\right)$. By the Mean Value Theorem we obtain that

$$
f(\alpha(t))-f(\alpha(0))=\left(d f_{1}\left(\alpha^{\prime}\left(t_{1}\right)\right) t, d f_{2}\left(\alpha^{\prime}\left(t_{2}\right)\right) t, d f_{3}\left(\alpha^{\prime}\left(t_{3}\right)\right) t\right)
$$

and that

$$
\alpha(t)-\alpha(0)=\left(\alpha_{1}^{\prime}\left(s_{1}\right) t, \alpha_{2}^{\prime}\left(s_{2}\right) t, \alpha_{3}^{\prime}\left(s_{3}\right) t\right)
$$

for small $t>0$ and some $t_{1}, t_{2}, t_{3}, s_{1}, s_{2}, s_{3} \in(0,1)$. Similar relations hold for the curve $\beta$ near 0 . 
Next, let $x=\alpha(t), y=\beta(\theta)$ and choose small $t$ and $\theta$ so that $|x|=|y|$. It follows that

$$
\begin{aligned}
\frac{|f(x)-f(0)|}{|f(y)-f(0)|}= & \frac{|f(x)| /|x|}{|f(y)| /|y|} \\
= & \frac{\frac{\left|\left(d f_{1}\left(\alpha^{\prime}\left(t_{1}\right)\right), d f_{2}\left(\alpha^{\prime}\left(t_{2}\right)\right), d f_{3}\left(\alpha^{\prime}\left(t_{3}\right)\right)\right)\right|}{\left|\left(\alpha_{1}^{\prime}\left(s_{1}\right) t, \alpha_{2}^{\prime}\left(s_{2}\right) t, \alpha_{3}^{\prime}\left(s_{3}\right) t\right)\right|}}{\frac{\left|\left(d f_{1}\left(\beta^{\prime}\left(t_{1}\right)\right), d f_{2}\left(\beta^{\prime}\left(t_{2}\right)\right), d f_{3}\left(\beta^{\prime}\left(t_{3}\right)\right)\right)\right|}{\left|\left(\beta_{1}^{\prime}\left(s_{1}\right) t, \beta_{2}^{\prime}\left(s_{2}\right) t, \beta_{3}^{\prime}\left(s_{3}\right) t\right)\right|}} .
\end{aligned}
$$

Since $f$ is 1-QC, letting $r=|x|=|y| \rightarrow 0$, (3.4) yields that

$$
1=\frac{\left|d f\left(\alpha^{\prime}(0)\right)\right| /\left|\alpha^{\prime}(0)\right|}{\left|d f\left(\beta^{\prime}(0)\right)\right| /\left|\beta^{\prime}(0)\right|}=\frac{\left|d f\left(e_{1}\right)\right|}{\left|d f\left(e_{2}\right)\right|}
$$

and hence condition (b) follows.

For the proof of $(\mathrm{b}) \Rightarrow(\mathrm{a})$, we fix $p \in \Sigma$. Since $f$ is differentiable, for any $x \in \Sigma$ in a neighborhood of $p$ we have

$$
f(x)-f(p)=d f_{p}\left(x^{\prime}-p\right)+|x-p| \epsilon(|x-p|),
$$

where $x^{\prime}$ is the orthogonal projection of $x$ on the tangent plane $T_{p}(\Sigma)$ and $\epsilon(|x-p|)$ is a vector in $\mathbb{R}^{3}$ with length approaching zero as $|x-p| \rightarrow 0$. Therefore, for small $r>0$ and $x, y \in \Sigma$ with $|x-p|=|y-p|=r$, it follows that

$$
\begin{aligned}
\frac{\left|d f_{p}\left(y^{\prime}-p\right)\right|-|y-p||\epsilon(|y-p|)|}{\left|d f_{p}\left(x^{\prime}-p\right)\right|+|x-p||\epsilon(|x-p|)|} & \leq \frac{|f(y)-f(p)|}{|f(x)-f(p)|} \\
& \leq \frac{\left|d f_{p}\left(y^{\prime}-p\right)\right|+|y-p||\epsilon(|y-p|)|}{\left|d f_{p}\left(x^{\prime}-p\right)\right|-|x-p||\epsilon(|x-p|)|}
\end{aligned}
$$

Letting $r \rightarrow 0$, (3.5) together with condition (b) yields that

$$
\limsup _{r \rightarrow 0} \frac{\sup \{|f(x)-f(p)|:|x-p|=r\}}{\inf \{|f(x)-f(p)|:|x-p|=r\}}=1
$$

and this shows that $f$ is 1 -QC at each point $p$.

The equivalence of conditions (b) and (c) is a standard result in linear algebra. A diffeomorphism $f$ satisfying condition (b) (or (c)) is usually called a conformal map in the language of differential geometry. The equivalence of (c) and (d) is also routine. But, due to the fact that we could not find an exact reference for this result, we include a proof here for completeness.

For the proof of $(\mathrm{c}) \Rightarrow(\mathrm{d})$, we use local coordinates $x=\left(x_{1}, x_{2}, x_{3}\right)$ at $p \in \Sigma$ and $y=\left(y_{1}, y_{2}, y_{3}\right)$ at $f(p) \in \Sigma_{1}$. Then

$$
d \sigma^{2}=d x_{1}^{2}+d x_{2}^{2}+d x_{3}^{2}, \quad d \sigma_{1}^{2}=d y_{1}^{2}+d y_{2}^{2}+d y_{3}^{2} .
$$

Fix any smooth curve $\alpha:(-1,1) \rightarrow \Sigma$ with $\alpha(0)=p$. Then the tangent vector

$$
v(t)=\alpha^{\prime}(t)=\left(\frac{d x_{1}}{d t}, \frac{d x_{2}}{d t}, \frac{d x_{3}}{d t}\right)
$$

is mapped to the tangent vector

$$
d f(v)=\left(\frac{d y_{1}}{d t}, \frac{d y_{2}}{d t}, \frac{d y_{3}}{d t}\right)
$$

by $d f$. Applying condition (c) to $v_{1}=v_{2}=v(t)$, we obtain that

$$
\left(\frac{d y_{1}}{d t}\right)^{2}+\left(\frac{d y_{2}}{d t}\right)^{2}+\left(\frac{d y_{3}}{d t}\right)^{2}=\lambda^{2}\left(\left(\frac{d x_{1}}{d t}\right)^{2}+\left(\frac{d x_{2}}{d t}\right)^{2}+\left(\frac{d x_{3}}{d t}\right)^{2}\right) .
$$


Since this is true for any smooth curve $\alpha$ as above, it yields that

$$
d y_{1}^{2}+d y_{2}^{2}+d y_{3}^{2}=\lambda^{2}\left(d x_{1}^{2}+d x_{2}^{2}+d x_{3}^{2}\right) .
$$

Thus (d) follows as desired. Conversely, if (d) holds, then (3.6) holds for any smooth curve. Applying (3.6) to a smooth curve with a unit tangent vector, we obtain (b). This completes the proof of Theorem 3.1.

\section{1-QC EMBEDDINGS OF PLANAR DOMAINS}

In this section we establish the existence of 1-QC maps of certain smooth surfaces onto planar domains. Let $\Sigma$ be a surface in $\mathbb{R}^{3}$ given by

$$
\Sigma=\{(x, y, \phi):(x, y) \in G, \phi=\phi(x, y)\},
$$

where $G$ is a domain in $\mathbb{R}^{2}$ and $\phi$ is a continuous function in $G$. We say that $\Sigma$ is a $C^{1+\alpha}$-smooth surface if $\phi$ has locally $\alpha$-Hölder continuous partial derivatives $\phi_{x}$ and $\phi_{y}$ for some $\alpha>0$.

Theorem 4.2. Let $\Sigma$ be a $C^{1+\alpha}$-smooth surface in $\mathbb{R}^{3}$ given by (4.1). Then $\Sigma$ can be mapped to a planar domain by a smooth 1-QC map.

Proof. Consider a map $F: \Sigma \rightarrow \mathbb{R}^{2}$ of the form

$$
F(x, y, \phi)=(u, v),
$$

where the functions $u=u(x, y)$ and $v=v(x, y)$ are to be determined so that the map $F$ is 1-QC on $\Sigma$. By Theorem 3.1, such a map is 1-QC if and only if the metrics on $\Sigma$ and its image domain $D$ satisfy that

$$
d u^{2}+d v^{2}=\lambda(x, y)^{2} d \sigma^{2}
$$

for some continuous function $\lambda(x, y)>0$ in $G$.

The metric $d \sigma^{2}$ can be computed as follows:

$$
\begin{aligned}
d \sigma^{2} & =d x^{2}+d y^{2}+d \phi^{2} \\
& =\left(1+\phi_{x}^{2}\right) d x^{2}+2 \phi_{x} \phi_{y} d x d y+\left(1+\phi_{y}^{2}\right) d y^{2} \\
& =g_{11} d x^{2}+2 g_{12} d x d y+g_{22} d y^{2},
\end{aligned}
$$

where

$$
g_{11}=1+\phi_{x}^{2}, g_{12}=\phi_{x} \phi_{y}, g_{22}=1+\phi_{y}^{2} .
$$

Using the complex notation $z=x+i y$, we deduce that

$$
\begin{aligned}
d \sigma^{2} & =\frac{1}{4} g_{11}(d z+d \bar{z})^{2}-\frac{i}{2} g_{12}\left(d z^{2}-d \bar{z}^{2}\right)-\frac{1}{4} g_{22}(d z-d \bar{z})^{2} \\
& =d z^{2}\left(\frac{1}{4} g_{11}-\frac{1}{4} g_{22}-\frac{i}{2} g_{12}\right)+d \bar{z}^{2}\left(\frac{1}{4} g_{11}-\frac{1}{4} g_{22}+\frac{i}{2} g_{12}\right)+d z d \bar{z}\left(\frac{1}{2} g_{11}+\frac{1}{2} g_{22}\right) \\
& =|A d z+B d \bar{z}|^{2}=|A|^{2}\left|d z+\frac{B}{A} d \bar{z}\right|^{2},
\end{aligned}
$$

where

$$
\begin{aligned}
& |A|^{2}=\frac{1}{2}\left[\frac{1}{2}\left(g_{11}+g_{22}\right)+\sqrt{g_{11} g_{22}-g_{12}^{2}}\right], \\
& \mu=\frac{B}{A}=\frac{\frac{1}{2}\left(g_{11}-g_{22}\right)+i g_{12}}{\frac{1}{2}\left(g_{11}+g_{22}\right)+\sqrt{g_{11} g_{22}-g_{12}^{2}}} .
\end{aligned}
$$


On the other hand, using the complex notation $w=u+i v$, the metric in $D$ can be written as

$$
d u^{2}+d v^{2}=|d w|^{2}=\left|w_{z}\right|^{2}\left|d z+\frac{w_{\bar{z}}}{w_{z}} d \bar{z}\right|^{2} .
$$

Hence, if the map $w:(x, y) \rightarrow(u, v)$ satisfies the Beltrami equation

$$
w_{\bar{z}}=\mu w_{z}
$$

with the coefficient $\mu$ given in (4.7), then the conformality condition (4.4) is satisfied with $\lambda(x, y)=\left|w_{z}\right| /|A|>0$.

It remains to show that the Beltrami equation (4.8) has a solution $w$ that is continuously differentiable in $G$ and whose complex dilatation is equal to $\mu$ everywhere in $G$. It follows from (4.6) and (4.7) that

$$
\begin{aligned}
|\mu|^{2} & =\frac{\frac{1}{2}\left(g_{11}+g_{22}\right)-\sqrt{g_{11} g_{22}-g_{12}^{2}}}{\frac{1}{2}\left(g_{11}+g_{22}\right)+\sqrt{g_{11} g_{22}-g_{12}^{2}}} \\
& =\frac{\frac{1}{2}\left(2+\phi_{x}^{2}+\phi_{y}^{2}\right)-\sqrt{1+\phi_{x}^{2}+\phi_{y}^{2}}}{\frac{1}{2}\left(2+\phi_{x}^{2}+\phi_{y}^{2}\right)+\sqrt{1+\phi_{x}^{2}+\phi_{y}^{2}}}<1
\end{aligned}
$$

for all $z=x+i y \in G$. Furthermore, since the partial derivatives $\phi_{x}$ and $\phi_{y}$ are locally $\alpha$-Hölder continuous in $G$, the Beltrami coefficient $\mu$ is also locally $\alpha$-Hölder continuous in $G$. By invoking Theorem 2.2, we conclude that the Beltrami equation (4.8) has a solution that is continuously differentiable and its complex dilatation coincides with the prescribed $\mu$ everywhere in $G$.

In conclusion, let $w=w(z)=u+i v$ be such a solution of (4.8) in $G$. Then the map given in (4.3) is a smooth 1-QC map from the surface $\Sigma$ onto the planar domain $D=w(G)$. This completes the proof of Theorem 4.2.

To conclude this section, we generalize Theorem 4.2 to the case where the surface is not necessarily the graph of a function.

Theorem 4.9. Let $\Sigma$ be a surface in $\mathbb{R}^{3}$ that admits a $C^{1+\alpha}$-parametrization by a domain $G$ in the plane:

$$
\Sigma=\{(p, q, t): p=p(x, y), q=q(x, y), t=t(x, y),(x, y) \in G\} .
$$

Then $\Sigma$ can be mapped to a planar domain by a smooth 1-QC map.

Proof. Similar to the proof of Theorem 4.2, a desired 1-QC map $F: \Sigma \rightarrow \mathbb{R}^{2}$ will be the composition of the inverse parametrization map: $(p, q, t) \rightarrow(x, y)$ and a quasiconformal map on $G$ whose complex dilatation is given by

$$
\mu=\frac{\frac{1}{2}\left(g_{11}-g_{22}\right)+i g_{12}}{\frac{1}{2}\left(g_{11}+g_{22}\right)+\sqrt{g_{11} g_{22}-g_{12}^{2}}},
$$

where

$$
g_{11}=p_{x}^{2}+q_{x}^{2}+t_{x}^{2}, \quad g_{12}=p_{x} p_{y}+q_{x} q_{y}+t_{x} t_{y}, \quad g_{22}=p_{y}^{2}+q_{y}^{2}+t_{y}^{2} .
$$

By Hölder's inequality,

$$
\begin{aligned}
g_{12}^{2} & =\left(p_{x} p_{y}+q_{x} q_{y}+t_{x} t_{y}\right)^{2} \\
& \leq\left(p_{x}^{2}+q_{x}^{2}+t_{x}^{2}\right)\left(p_{y}^{2}+q_{y}^{2}+t_{y}^{2}\right)=g_{11} g_{22}
\end{aligned}
$$


with equality throughout if and only if

$$
\left(p_{x}, q_{x}, t_{x}\right)=r(x, y)\left(p_{y}, q_{y}, t_{y}\right)
$$

for some $r$. However, since the map $(x, y) \rightarrow(p, q, t)$ is a diffeomorphism, the linear map between tangent planes:

$$
\left(\begin{array}{cc}
p_{x} & p_{y} \\
q_{x} & q_{y} \\
t_{x} & t_{y}
\end{array}\right): T(G) \rightarrow T(\Sigma)
$$

has rank 2. Therefore, the strict inequality $g_{11} g_{22}-g_{12}^{2}>0$ holds for all $(x, y) \in G$. Hence it follows that

$$
|\mu|^{2}=\frac{\frac{1}{2}\left(g_{11}+g_{22}\right)-\sqrt{g_{11} g_{22}-g_{12}^{2}}}{\frac{1}{2}\left(g_{11}+g_{22}\right)+\sqrt{g_{11} g_{22}-g_{12}^{2}}}<1
$$

for all $(x, y) \in G$. Thus, by invoking Theorem 2.2 again, we conclude that a desired solution to the Beltrami equation exists in $G$. This completes the proof of Theorem 4.9.

\section{1-QC EMBEDDINGS OF THE UNIT SPHERE}

After considering surfaces parametrized by open domains in the plane, we now turn our attention to topological spheres and establish another main result of this paper.

Theorem 5.1. Any surface $\Sigma \subset \mathbb{R}^{3}$ that is $C^{1+\alpha}$-diffeomorphic to the unit sphere $\mathbb{S}^{2}$ can be mapped onto $\mathbb{S}^{2}$ by a 1-QC map.

5.2. The stereographic projection $\pi$. Before proving Theorem 5.1, we recall the stereographic projection $\pi: \mathbb{R}^{2} \rightarrow \mathbb{S}^{2} \backslash\{\mathbb{N}\}$ given by

$$
\pi(x, y)=\left(\frac{2 x}{x^{2}+y^{2}+1}, \frac{2 y}{x^{2}+y^{2}+1}, \frac{x^{2}+y^{2}-1}{x^{2}+y^{2}+1}\right),
$$

where $\mathbb{N}=(0,0,1) \in \mathbb{S}^{2}$ is the north pole. If we denote the coordinates on $\mathbb{S}^{2}$ by $(u, v, w)$, then the metric on $\mathbb{S}^{2}$ is given by

$$
d s^{2}=d u^{2}+d v^{2}+d w^{2}=\frac{4}{\left(x^{2}+y^{2}+1\right)^{2}}\left(d x^{2}+d y^{2}\right) .
$$

Thus $\pi: \mathbb{R}^{2} \rightarrow \mathbb{S}^{2} \backslash\{\mathbb{N}\}$ is conformal in the Euclidean metric of $\mathbb{R}^{2}$ and $\pi: \overline{\mathbb{R}}^{2} \rightarrow \mathbb{S}^{2}$ is conformal in the chordal metric of $\overline{\mathbb{R}}^{2}$.

5.4. Proof of Theorem 5.1. Let $g: \mathbb{S}^{2} \rightarrow \Sigma$ be a $C^{1+\alpha}$-diffeomorphism and denote the coordinates on $\Sigma$ by $(p, q, t)$. Then the desired 1-QC map $F: \Sigma \rightarrow \mathbb{S}^{2}$ will be the following composition

$$
F(p, q, t)=\pi \circ f \circ \pi^{-1} \circ g^{-1}(p, q, t): \Sigma \rightarrow \mathbb{S}^{2},
$$

where $f=w(z)=u+i v: \overline{\mathbb{R}}^{2} \rightarrow \overline{\mathbb{R}}^{2}$ is a solution to a Beltrami equation

$$
w_{\bar{z}}=\mu w_{z}
$$

whose coefficient $\mu$ is to be determined. We will use Theorem 3.1 and the metric condition (d) there to show that such a map is indeed 1-QC. 
5.6. Comparison of the metrics. To compare the metric $d \sigma^{2}$ on $\Sigma$ and the metric $d s^{2}$ on $\mathbb{S}^{2}$ off the north pole under the correspondence $(5.5)$, we note that $\Sigma$ has the following parametrization

$$
\Sigma=\left\{(p, q, t)=g \circ \pi(x, y):(x, y) \in \overline{\mathbb{R}}^{2}\right\} .
$$

Thus, as in the previous section, one deduces that

$$
\begin{aligned}
d \sigma^{2} & =d p^{2}+d q^{2}+d t^{2} \\
& =\left(p_{x}^{2}+q_{x}^{2}+t_{x}^{2}\right) d x^{2}+2\left(p_{x} p_{y}+q_{x} q_{y}+t_{x} t_{y}\right) d x d y+\left(p_{y}^{2}+q_{y}^{2}+t_{y}^{2}\right) d y^{2} \\
& =g_{11} d x^{2}+2 g_{12} d x d y+g_{22} d y^{2} \\
& =|A|^{2}|d z+\mu d \bar{z}|^{2},
\end{aligned}
$$

where $z=x+i y, A$ and $\mu$ are as in (4.7), and

$$
g_{11}=p_{x}^{2}+q_{x}^{2}+t_{x}^{2}, \quad g_{12}=p_{x} p_{y}+q_{x} q_{y}+t_{x} t_{y}, \quad g_{22}=p_{y}^{2}+q_{y}^{2}+t_{y}^{2} .
$$

On the other hand, the metric on $\mathbb{S}^{2} \backslash\{\mathbb{N}\}$ is given by

$$
d s^{2}=\frac{4}{\left(u^{2}+v^{2}+1\right)^{2}}\left(d u^{2}+d v^{2}\right)=\frac{4\left|w_{z}\right|^{2}}{\left(u^{2}+v^{2}+1\right)^{2}}\left|d z+\frac{w_{\bar{z}}}{w_{z}} d \bar{z}\right|^{2} .
$$

Therefore, by comparing $d s^{2}$ on $\mathbb{S}^{2}$ and $d \sigma^{2}$ on $\Sigma$, we see that the map $F$ : $\Sigma \backslash F^{-1}(\{\mathbb{N}\}) \rightarrow \mathbb{S}^{2} \backslash\{\mathbb{N}\}$ satisfies the conformality condition (d) in Theorem 3.1 if and only if $f=w(z)$ is a $C^{1}$-smooth solution to the Beltrami equation with coefficient

$$
\mu=\frac{\frac{1}{2}\left(g_{11}-g_{22}\right)+i g_{12}}{\frac{1}{2}\left(g_{11}+g_{22}\right)+\sqrt{g_{11} g_{22}-g_{12}^{2}}}
$$

and $g_{i j}$ given by (5.7).

5.9. Estimate of $|\mu|$. Our goal here is to show that there is a constant $c<1$ such that $|\mu| \leq c$ for all $(x, y) \in \overline{\mathbb{R}}^{2}$. For this we recall, as computed in the proof of Theorem 4.9, that

$$
|\mu|^{2}=\frac{\left(g_{11}+g_{22}\right)-2 \sqrt{g_{11} g_{22}-g_{12}^{2}}}{\left(g_{11}+g_{22}\right)+2 \sqrt{g_{11} g_{22}-g_{12}^{2}}}<1
$$

for all $(x, y) \in \mathbb{R}^{2}$. Therefore, it suffices to show that, as $x^{2}+y^{2} \rightarrow \infty$, the limit of $|\mu|$ is strictly less than one. For this, we only need to show that

$$
\lim _{x^{2}+y^{2} \rightarrow \infty} \frac{\sqrt{g_{11} g_{22}-g_{12}^{2}}}{g_{11}+g_{22}}>0 .
$$

Write the diffeomorphism $g: \mathbb{S}^{2} \rightarrow \Sigma$ as $(p, q, t)=g(\alpha, \beta, \gamma)$. Then the parametrization of $\Sigma$ by $\overline{\mathbb{R}}^{2}$ is given by

$$
g \circ \pi:(x, y) \in \overline{\mathbb{R}}^{2} \rightarrow(\alpha, \beta, \gamma) \in \mathbb{S}^{2} \rightarrow(p, q, t) \in \Sigma .
$$

By chain rule of differentials,

$$
\frac{\partial(p, q, t)}{\partial(x, y)}=\frac{\partial(p, q, t)}{\partial(\alpha, \beta, \gamma)} \cdot \frac{\partial(\alpha, \beta, \gamma)}{\partial(x, y)}
$$


which yields that

(5.11)

$$
\left(\begin{array}{cc}
p_{x} & p_{y} \\
q_{x} & q_{y} \\
t_{x} & t_{y}
\end{array}\right)=\frac{2}{\left(x^{2}+y^{2}+1\right)^{2}}\left(\begin{array}{ccc}
p_{\alpha} & p_{\beta} & p_{\gamma} \\
q_{\alpha} & q_{\beta} & q_{\gamma} \\
t_{\alpha} & t_{\beta} & t_{\gamma}
\end{array}\right)\left(\begin{array}{cc}
-x^{2}+y^{2}+1 & -2 x y \\
-2 x y & x^{2}-y^{2}+1 \\
2 x & 2 y
\end{array}\right) .
$$

Recall that $g_{i j}$ are defined in terms of the partials of $p, q, t$ in (5.7). Thus, to estimate the quotient in (5.10), one can ignore the factor $2 /\left(x^{2}+y^{2}+1\right)^{2}$ in $(5.11)$ since it will cancel out. In doing so, we let

$$
G_{i j}=\frac{\left(x^{2}+y^{2}+1\right)^{4}}{4} g_{i j}
$$

Then we have

$$
\frac{\sqrt{g_{11} g_{22}-g_{12}^{2}}}{g_{11}+g_{22}}=\frac{\sqrt{G_{11} G_{22}-G_{12}^{2}}}{G_{11}+G_{22}}
$$

and

$$
\begin{aligned}
G_{11} & =\left(\left(-x^{2}+y^{2}+1\right) p_{\alpha}-2 x y p_{\beta}+2 x p_{\gamma}\right)^{2} \\
& +\left(\left(-x^{2}+y^{2}+1\right) q_{\alpha}-2 x y q_{\beta}+2 x q_{\gamma}\right)^{2} \\
& +\left(\left(-x^{2}+y^{2}+1\right) t_{\alpha}-2 x y t_{\beta}+2 x t_{\gamma}\right)^{2} .
\end{aligned}
$$

Next, since $(p, q, t)=g(\alpha, \beta, \gamma)$ is a diffeomorphism, $\operatorname{rank}(D g) \geq 2$. By rotating the sphere if necessary, we may assume that

$$
\operatorname{Rank}\left(\begin{array}{cc}
p_{\alpha} & p_{\beta} \\
q_{\alpha} & q_{\beta} \\
t_{\alpha} & t_{\beta}
\end{array}\right)=2
$$

Thus $p_{\alpha}^{2}+q_{\alpha}^{2}+t_{\alpha}^{2}>0$ and $p_{\beta}^{2}+q_{\beta}^{2}+t_{\beta}^{2}>0$ on $\mathbb{S}^{2}$. It follows from (5.13) that, as $x^{2}+y^{2} \rightarrow \infty$, the dominating term in $G_{11}$ (denoted again by $G_{11}$ ) is

$$
\begin{aligned}
G_{11}= & \left(\left(-x^{2}+y^{2}+1\right) p_{\alpha}-2 x y p_{\beta}\right)^{2}+\left(\left(-x^{2}+y^{2}+1\right) q_{\alpha}-2 x y q_{\beta}\right)^{2} \\
& +\left(\left(-x^{2}+y^{2}+1\right) t_{\alpha}-2 x y t_{\beta}\right)^{2} \\
= & \left(-x^{2}+y^{2}+1\right)^{2}\left(p_{\alpha}^{2}+q_{\alpha}^{2}+t_{\alpha}^{2}\right)+(2 x y)^{2}\left(p_{\beta}^{2}+q_{\beta}^{2}+t_{\beta}^{2}\right) \\
& -4 x y\left(-x^{2}+y^{2}+1\right)\left(p_{\alpha} p_{\beta}+q_{\alpha} q_{\beta}+t_{\alpha} t_{\beta}\right) .
\end{aligned}
$$

To obtain the dominating term in $G_{22}$, we only need to interchange $x$ with $y$ and $\alpha$ with $\beta$ in the expression of $G_{11}$ above. Therefore, after further cancellation and discarding minor terms, we obtain the following dominating term in $G_{11}+G_{22}$ :

$$
\begin{aligned}
G_{11}+G_{22}= & \left(\left(-x^{2}+y^{2}+1\right)^{2}+(2 x y)^{2}\right)\left(p_{\alpha}^{2}+q_{\alpha}^{2}+t_{\alpha}^{2}\right) \\
& +\left(\left(x^{2}-y^{2}+1\right)^{2}+(2 x y)^{2}\right)\left(p_{\beta}^{2}+q_{\beta}^{2}+t_{\beta}^{2}\right) \\
= & \left(\left(x^{2}+y^{2}+1\right)^{2}-4 x^{2}\right)\left(p_{\alpha}^{2}+q_{\alpha}^{2}+t_{\alpha}^{2}\right) \\
& +\left(\left(x^{2}+y^{2}+1\right)^{2}-4 y^{2}\right)\left(p_{\beta}^{2}+q_{\beta}^{2}+t_{\beta}^{2}\right) \\
\approx & \left(x^{2}+y^{2}+1\right)^{2}\left(\left(p_{\alpha}^{2}+q_{\alpha}^{2}+t_{\alpha}^{2}\right)+\left(p_{\beta}^{2}+q_{\beta}^{2}+t_{\beta}^{2}\right)\right) .
\end{aligned}
$$

Tedious but similar computation yields that, as $x^{2}+y^{2} \rightarrow \infty$, the dominating term of $G_{11} G_{22}-G_{12}^{2}$ is

$$
\begin{aligned}
G_{11} G_{22}-G_{12}^{2}= & \left(\left(x^{2}+y^{2}\right)^{2}-1\right)^{2}\left[\left(p_{\alpha}^{2}+q_{\alpha}^{2}+t_{\alpha}^{2}\right)\left(p_{\beta}^{2}+q_{\beta}^{2}+t_{\beta}^{2}\right)\right. \\
& \left.-\left(p_{\alpha} p_{\beta}+q_{\alpha} q_{\beta}+t_{\alpha} t_{\beta}\right)^{2}\right] .
\end{aligned}
$$


Hence it follows that

$$
\begin{aligned}
\lim _{x^{2}+y^{2} \rightarrow \infty} \frac{\sqrt{g_{11} g_{22}-g_{12}^{2}}}{g_{11}+g_{22}} & =\lim _{x^{2}+y^{2} \rightarrow \infty} \frac{\sqrt{G_{11} G_{22}-G_{12}^{2}}}{G_{11}+G_{22}} \\
& =\lim _{x^{2}+y^{2} \rightarrow \infty} \frac{\left(\left(x^{2}+y^{2}\right)^{2}-1\right) \sqrt{\mathbf{P}_{\alpha}^{2} \mathbf{P}_{\beta}^{2}-\left(\mathbf{P}_{\alpha} \mathbf{P}_{\beta}\right)^{2}}}{\left(x^{2}+y^{2}+1\right)^{2}\left(\mathbf{P}_{\alpha}^{2}+\mathbf{P}_{\beta}^{2}\right)} \\
& =\frac{\sqrt{\mathbf{P}_{\alpha}^{2} \mathbf{P}_{\beta}^{2}-\left(\mathbf{P}_{\alpha} \mathbf{P}_{\beta}\right)^{2}}}{\left(\mathbf{P}_{\alpha}^{2}+\mathbf{P}_{\beta}^{2}\right)}
\end{aligned}
$$

where

$$
\mathbf{P}_{\alpha}^{2}=p_{\alpha}^{2}+q_{\alpha}^{2}+t_{\alpha}^{2}, \mathbf{P}_{\beta}^{2}=p_{\beta}^{2}+q_{\beta}^{2}+t_{\beta}^{2}, \mathbf{P}_{\alpha} \mathbf{P}_{\beta}=p_{\alpha} p_{\beta}+q_{\alpha} q_{\beta}+t_{\alpha} t_{\beta},
$$

and the last expression in (5.18) is evaluated at the north pole and has a positive value because of (5.14). This shows that the Beltrami coefficient $\mu$ defined in (5.8) has the desired property as claimed in 5.9.

5.20. Solution to the Beltrami equation. Since the map $g: \mathbb{S}^{2} \rightarrow \Sigma$ is $C^{1+\alpha_{-}}$ smooth, the coefficient $\mu$ in (5.8) is $\alpha$-Hölder continuous. Therefore, by the classical measurable Riemann Mapping Theorem and [LV, Theorem 7.2, p. 235], there is a normalized $C^{1}$-smooth solution $f=w(z): \overline{\mathbb{R}}^{2} \rightarrow \overline{\mathbb{R}}^{2}$ to the Beltrami equation $w_{\bar{z}}=\mu w_{z}$ such that $\left|w_{z}\right|$ is positive and finite in $\overline{\mathbb{R}}^{2}$ and that $|w| /|z| \rightarrow 1$ as $|z| \rightarrow \infty$.

5.21. 1-Quasiconformality of the map $F$. Let $f=w(z)$ be the solution to the Beltrami equation described above. To show that the map $F: \Sigma \rightarrow \mathbb{S}^{2}$ defined in (5.5) is 1-QC (or conformal), we need to verify the metric condition (d) in Theorem 3.1. From the computation in subsection 5.6, we obtain that

$$
\frac{d \sigma^{2}}{d s^{2}}=\lambda^{2}(x, y)
$$

for all $(p, q, t) \in \Sigma$, where

$$
\lambda^{2}(x, y)=\frac{|A|^{2}\left(u^{2}+v^{2}+1\right)^{2}}{4\left|w_{z}\right|^{2}}
$$

for $(x, y) \in \mathbb{R}^{2}$,

$$
\lambda(\infty)=\lim _{x^{2}+y^{2} \rightarrow \infty} \frac{|A|^{2}\left(u^{2}+v^{2}+1\right)^{2}}{4\left|w_{z}\right|^{2}}
$$

and

$$
|A|^{2}=\frac{1}{4}\left[\left(g_{11}+g_{22}\right)+2 \sqrt{g_{11} g_{22}-g_{12}^{2}}\right]
$$

as in (4.7) with $g_{i j}$ given in (5.7). Since all the maps involved in (5.5) are diffeomorphisms on the defined surfaces or extended planes, we see that $0<\lambda^{2}(x, y)<\infty$ for all $(x, y) \in \mathbb{R}^{2}$. Thus, it remains to show that $\lambda^{2}(x, y)$ has a finite positive limit as $x^{2}+y^{2} \rightarrow \infty$. To this end, due to the properties of the solution $f=w(z)$ noted in 5.20, we only need to show that $|A|^{2}\left(x^{2}+y^{2}+1\right)^{2}$ has a finite positive limit as 
$x^{2}+y^{2} \rightarrow \infty$. We note that

$$
\begin{aligned}
|A|^{2}\left(x^{2}+y^{2}+1\right)^{2} & =\frac{\left(x^{2}+y^{2}+1\right)^{2}}{4}\left[\left(g_{11}+g_{22}\right)+2 \sqrt{g_{11} g_{22}-g_{12}^{2}}\right] \\
& =\frac{G_{11}+G_{22}}{\left(x^{2}+y^{2}+1\right)^{2}}\left[1+\frac{2 \sqrt{G_{11} G_{22}-G_{12}^{2}}}{G_{11}+G_{22}}\right] .
\end{aligned}
$$

Thus it follows from (5.16) and (5.18) that

$$
\lim _{x^{2}+y^{2} \rightarrow \infty}|A|^{2}\left(x^{2}+y^{2}+1\right)^{2}=\left(\mathbf{P}_{\alpha}^{2}+\mathbf{P}_{\beta}^{2}\right)\left[1+\frac{2 \sqrt{\mathbf{P}_{\alpha}^{2} \mathbf{P}_{\beta}^{2}-\left(\mathbf{P}_{\alpha} \mathbf{P}_{\beta}\right)^{2}}}{\left(\mathbf{P}_{\alpha}^{2}+\mathbf{P}_{\beta}^{2}\right)}\right],
$$

which, evaluated at the north pole, is finite and positive. This shows that the map $F: \Sigma \rightarrow \mathbb{S}^{2}$ given in (5.5) satisfies the condition (d) in Theorem 3.1, and hence 1-QC. The proof of Theorem 5.1 is complete.

\section{EXAMPLES}

In this section, we give several examples to illustrate the results in Theorems 4.2 and 5.1. Recall that Theorem 4.2 deals with surfaces $\Sigma$ given by the graph of a smooth function $\phi(x, y)$ over a domain $G \subset \mathbb{R}^{2}$. According to the calculations in the proof of Theorem 4.2, the corresponding Beltrami coefficient associated with $\phi$ is given by

$$
\mu=\frac{\left(\phi_{x}^{2}-\phi_{y}^{2}\right)+2 i \phi_{x} \phi_{y}}{2+\phi_{x}^{2}+\phi_{y}^{2}+2 \sqrt{1+\phi_{x}^{2}+\phi_{y}^{2}}}
$$

with

$$
|\mu|=\frac{\sqrt{1+\phi_{x}^{2}+\phi_{y}^{2}}-1}{\sqrt{1+\phi_{x}^{2}+\phi_{y}^{2}}+1} .
$$

Thus, when $\phi_{x}^{2}+\phi_{y}^{2}$ is bounded in $G$, the corresponding Beltrami equation can be solved by using the classical Riemann mapping theorem. But when $\phi_{x}^{2}+\phi_{y}^{2}$ is unbounded in $G$, Theorem 2.2 is needed in solving the Beltrami equation. We first give two simple examples with bounded partials.

6.3. Example. Consider the surface $\Sigma$ given by the graph of the function $\phi(x, y)=$ $\sin x$. Then the corresponding Beltrami coefficient calculates to

$$
\mu=\frac{\cos ^{2} x}{2+\cos ^{2} x+2 \sqrt{1+\cos ^{2} x}},
$$

which admits the following estimates:

$$
|\mu|=\frac{\sqrt{1+\cos ^{2} x}-1}{\sqrt{1+\cos ^{2} x}+1} \leq(\sqrt{2}-1)^{2}<1 .
$$

A desired 1-QC map $F: \Sigma \rightarrow \mathbb{R}^{2}$ is the composition of the projection $(x, y, \phi) \rightarrow$ $(x, y)$ and a quasiconformal map of the plane whose complex dilatation is the above $\mu$. 
6.4. Example. Let $\Sigma$ be a tilted plane given by $\phi(x, y)=a x$ for some constant $a>0$. Then the Beltrami coefficient is

$$
\mu=\frac{\sqrt{1+a^{2}}-1}{\sqrt{1+a^{2}}+1}
$$

In this case the 1-QC map $F: \Sigma \rightarrow \mathbb{R}^{2}$ is given by $F(x, y, \phi)=\left(\sqrt{1+a^{2}} x, y\right)$, which is just a rotation about the $y$-axis as expected.

6.5. Paraboloid. Next we consider an example with unbounded partials, namely, the paraboloid determined by $\phi(x, y)=x^{2}+y^{2}$ over $\mathbb{R}^{2}$. Then the Beltrami coefficient is

$$
\mu=\frac{2 x^{2}-2 y^{2}+i 4 x y}{1+2 x^{2}+2 y^{2}+\sqrt{1+4 x^{2}+4 y^{2}}}
$$

with the distortion function given by

$$
K(x, y)=\frac{1+|\mu|}{1-|\mu|}=\sqrt{1+4 x^{2}+4 y^{2}} .
$$

According to Example 2.8, the 1-QC map $F: \Sigma \rightarrow \mathbb{R}^{2}$ claimed in Theorem 4.2 may not be onto for the paraboloid. But, nevertheless, a paraboloid is 1-QC equivalent to a planar domain, which is somewhat surprising.

6.6. Ellipsoid. Finally, we use the example of an ellipsoid to illustrate the result in Theorem 5.1. Consider the ellipsoid

$$
\Sigma=\left\{(p, q, t) \in \mathbb{R}^{3}: \frac{p^{2}}{a^{2}}+\frac{q^{2}}{a^{2}}+t^{2}=1\right\},
$$

where $a \geq 1$ is a constant. Then $\Sigma$ is diffeomorphic to the unit sphere $\mathbb{S}^{2}$ under the map $g: \mathbb{S}^{2} \rightarrow \Sigma$ given by $g(\alpha, \beta, \gamma)=(a \alpha, a \beta, \gamma)$. By Theorem 5.1, $\Sigma$ and $\mathbb{S}^{2}$ are 1-QC equivalent. To find such a 1-QC map, according to the proof of Theorem 5.1 , one needs to solve a Beltrami equation in $\overline{\mathbb{C}}$ whose coefficient is given by

$$
\mu=\frac{-2\left(a^{2}-1\right)\left(x^{2}-y^{2}\right)-4 i\left(a^{2}-1\right) x y}{a^{2}\left[\left(x^{2}+y^{2}\right)^{2}+1\right]+2\left(x^{2}+y^{2}\right)+a\left(x^{2}+y^{2}+1\right) \sqrt{a^{2}\left(x^{2}+y^{2}-1\right)^{2}+4\left(x^{2}+y^{2}\right)}} .
$$

A normalized smooth solution to such an equation is guaranteed to exist $\mathrm{LV}$, Theorem 7.2, p. 235]. But it seems to be no easy task to find such a solution explicitly. It would be interesting to find an explicit 1-QC map between the ellipsoid $\Sigma$ and the unit sphere $\mathbb{S}^{2}$.

6.7. Open problems. We close this paper with some open problems.

(a) According to Theorem 4.2, a paraboloid such as the one considered in 6.5 can be mapped onto a planar domain by a 1-QC map. Find such a map explicitly.

(b) Similarly, find an explicit 1-QC map between the unit sphere and an ellipsoid.

(c) Characterize all topological spheres in $\mathbb{R}^{3}$ that are 1-QC equivalent to the unit sphere. Theorem 5.1 shows that $C^{1+\alpha}$-smooth topological spheres are 1-QC equivalent to the unit sphere. Bonk and Kleiner [BK] characterized all topological spheres that are quasisymmetrically equivalent to the unit sphere. It would be interesting to explore the connections between these two. 


\section{ACKNOWLEDGMENT}

The author would like to thank Tadeusz Iwaniec and Kari Astala for valuable discussions on regularity of solutions to the Beltrami equation with degenerate coefficient.

\section{REFERENCES}

[Be] L. Bers, Riemann surfaces, New York University, New York, 1957-1958.

[BK] M. Bonk and B. Kleiner, Quasisymmetric parametrizations of two-dimensional metric spheres, Invent. Math. 150 (2002), 127-183. MR1930885 (2004k:53057)

[Ca] M.P. do Carmo, Differential geometry of curves and surfaces, Prentice-Hall, New Jersey, 1976. MR.0394451 (52:15253)

[Ge1] F.W. Gehring, Rings and quasiconformal mappings in space, Trans. Amer. Math. Soc. 103 (1962), 353-393. MR0139735 (25:3166)

[Ge2] F.W. Gehring, Quasiconformal mappings in Euclidean spaces, Elsevier, Amsterdam, 2005, Handbook of complex analysis: Geometric function theory 2, 1-29. MR2121856 (2005k:30044)

[He] J. Heinonen, Lectures on analysis on metric spaces, Springer, New York, 2001. MR 1800917 (2002c:30028)

[IM1] T. Iwaniec and G.J. Martin, Geometric function theory and non-linear analysis, Clarendon Press, Oxford, 2001. MR 1859913 (2003c:30001)

[IM2] T. Iwaniec and G.J. Martin, The Beltrami Equations, to appear, Memoirs of the AMS.

[Le] O. Lehto, Univalent functions and Teichmüller spaces, Springer, New York, 1987. MR867407 (88f:30073)

[LV] O. Lehto and K. Virtanen, Quasiconformal mappings in the plane, Springer-Verlag, New York, 1973. MR0344463 (49:9202)

[Mo] G.D. Mostow, Quasi-conformal mappings in $n$-space and the rigidity of hyperbolic space forms, Inst. Hautes Etudes Sci. Publ. Math. 34 (1968), 53-104. MR0236383 (38:4679)

[Re1] Yu.G. Reshetnyak, Liouville's theorem on conformal mappings for minimal regularity assumptions, Sibirsk. Mat. Zh. 8 (1967), 835-840. MR0218544 (36:1630)

[Re2] Yu.G. Reshetnyak, On stability bounds in the Liouville theorem on conformal mappings of multidimensional spaces, Sibirsk. Mat. Zh. 11 (1970), 1121-1139. MR0269821 (42:4716)

Department of Mathematics and Computer Science, Emory University, Atlanta, GeOrgia 30322

E-mail address: syang@mathcs.emory.edu 